\title{
Comparative clinicopathological characteristics of colon and rectal $\mathrm{T} 1$ carcinoma
}

\author{
KATSURO ICHIMASA, SHIN-EI KUDO, HIDEYUKI MIYACHI, YUTA KOUYAMA, TAKEMASA HAYASHI, \\ KUNIHIKO WAKAMURA, TOMOKAZU HISAYUKI, TOYOKI KUDO, MASASHI MISAWA, \\ YUICHI MORI, SHINGO MATSUDAIRA, EIJI HIDAKA, SHIGEHARU HAMATANI and FUMIO ISHIDA
}

Digestive Disease Center, Showa University Northern Yokohama Hospital, Yokohama 224-8503, Japan

Received August 20, 2015; Accepted October 7, 2016

DOI: $10.3892 / 01.2016 .5464$

\begin{abstract}
Lymph node metastasis significantly influences the management of patients with colorectal carcinoma. It has been observed that the biology of colorectal carcinoma differs by location. The aim of the current study was to retrospectively compare the clinicopathological characteristics of patients with colon and rectal T1 carcinomas, particularly their rates of lymph node metastasis. Of the 19,864 patients who underwent endoscopic or surgical resection of colorectal neoplasms at Showa University Northern Yokohama Hospital, 557 had T1 surgically resected carcinomas, including 457 patients with colon T1 carcinomas and 100 patients with rectal T1 carcinomas. Analysed clinicopathological features included patient age, gender, tumor size, morphology, tumor budding, invasion depth, vascular invasion, histological grade, lymphatic invasion and lymph node metastasis. Rectal T1 carcinomas were significantly larger than colon T1 carcinomas (mean \pm standard deviation: $23.7 \pm 13.1 \mathrm{~mm}$ vs. $19.9 \pm 11.0 \mathrm{~mm}, \mathrm{P}<0.01)$ and were accompanied by significantly higher rates of vascular invasion $(48.0 \%$ vs. $30.2 \%$, $\mathrm{P}<0.01)$. Significant differences were not observed among any other clinicopathological factors. In conclusion, tumor location itself was not a risk factor for lymph node metastasis in colorectal T1 carcinomas, even though on average, rectal T1 carcinomas were larger and accompanied by a significantly higher rate of vascular invasion than colon T1 carcinomas.
\end{abstract}

\section{Introduction}

Colorectal cancer is one of the most common types of cancer in the world (1). In 2008, colorectal cancer was the leading cause of cancer-associated mortality in Japanese women and the third

Correspondence to: Professor Shin-Ei Kudo, Digestive Disease Center, Showa University Northern Yokohama Hospital, 35-1 Chigasaki-chuo, Tsuzuki-ku, Yokohama 224-8503, Japan

E-mail:kudos@med.showa-u.ac.jp

Key words: clinicopathological features, colorectal surgery, colorectal T1 carcinoma, endoscopic resection, lymph node metastasis leading cause in Japanese men (2). The biology of colorectal carcinoma may differ by location and it remains unclear whether carcinoma of the colon (caecum to sigmoid) and rectum should be considered as a single entity or as two distinct entities (3).

There are two major arguments for dividing colorectal carcinoma according to location. Firstly, rectal carcinoma may have a more malignant nature than colon carcinoma; it is more likely to metastasize to the lymph nodes or to recur (4). Furthermore, the risk of local recurrence following endoscopic resection has been reported to be significantly higher for rectal T1 carcinoma than for colon T1 carcinoma (5). Secondly, rectal surgery is more invasive than colon surgery, with higher rates of post-operative complications, including anastomotic leakage (6). In addition, a number of patients with rectal cancer are left with permanent stomas following standard abdominoperineal resection, and low anterior resection may lead to disorders and complications of anal functions, thus reducing patient quality of life (7).

In general, lymph node metastasis occurs in approximately $10 \%$ of patients with colorectal $\mathrm{T} 1$ carcinoma $(8,9)$, therefore, it is essential to thoroughly assess the need for surgical resection in such patients. The present study was designed to compare the clinicopathological characteristics of patients with colon and rectal T1 carcinoma, and to determine whether these carcinomas should be considered as a single entity or two distinct entities when deciding on surgical treatment.

\section{Patients and methods}

Patients. The study cohort included 557 patients with T1 colorectal carcinoma who were treated at Showa University Northern Yokohama Hospital (Yokohama, Japan) between April 2001 and March 2013. Patients were eligible for inclusion if they underwent endoscopic resection followed by additional surgery or surgical resection as a first-line treatment. Patients were excluded if they underwent endoscopic resection alone; exhibited evidence of inflammatory bowel disease, familial adenomatous polyposis or Lynch syndrome; had synchronous or metachronous advanced colorectal carcinoma or exhibited evidence of malignant disease in any other organ. None of the patients included in the study had received preoperative radiotherapy or neoadjuvant chemotherapy.

The rectum was defined as the area between the upper border of the anal canal and the lower border of the second 
sacral vertebra. Patient characteristics that were analysed included age, gender, tumor size, morphology, histological type, tumor budding, invasion depth, intramural lymphatic invasion, intramural vascular invasion and lymph node metastasis.

In a subanalysis, the sizes of colon and rectal $\mathrm{T} 1$ carcinomas were evaluated according to their morphologies. Tumor morphology was classified into three types according to the Paris endoscopic categorization of superficial neoplastic lesions (10): Depressed type; slightly elevated and flat type, including laterally spreading tumors (LSTs); and protruded type. LSTs were further divided into granular type (LST-G) and nongranular type (LST-NG) (11).

The protocol of the current study was approved by the ethics committee of Showa University Northern Yokohama Hospital (no. 1201-05) and registered in the UMIN Clinical Trials Registry (UMIN000010979). Written informed consent was obtained from all patients prior to treatment.

Endoscopic procedure. Examinations were performed using magnifying colonoscopies (CF-H260AZI or PCF-Q240ZI; Olympus Optical Corporation, Tokyo, Japan), which enhance images 75- to 100-fold. All colorectal lesions were evaluated in real-time under magnifying chromoendoscopy in the presence of $0.40 \%$ indigo carmine (Nagase Medicals Co., Ltd., Hyogo, Japan) to determine pit pattern classification $(12,13)$, and tumors with a type $\mathrm{V}$ pit pattern were evaluated using $0.05 \%$ crystal violet (Koso Chemical Co., Ltd., Gyoda, Japan) (13). Lesions observed to have III, IV, or $\mathrm{V}_{\mathrm{I}}$ low-grade pit patterns (i.e., adenomas, intramucosal colorectal carcinomas and slightly invasive submucosal colorectal carcinomas) were resected endoscopically. Patients with lesions exhibiting a $\mathrm{V}_{\mathrm{I}}$ high-grade or $\mathrm{V}_{\mathrm{N}}$ pit pattern (i.e., massively invasive submucosal colorectal carcinomas) were referred for surgery. Patients who refused surgery underwent endoscopic resection as a first-line treatment.

Surgical procedure. Patients who were endoscopically diagnosed with massively invasive submucosal carcinoma and agreed to undergo surgical resection underwent curative resection with lymph node dissection, either laparoscopically or using an open procedure. Patients were considered to be at high risk for lymph node metastasis if they exhibited vertically positive margins, an unfavourable histological type, evidence of vascular or lymphatic invasion, high-grade tumor budding or massively invasive carcinoma following endoscopic resection. Additional curative surgery with lymph node dissection was recommended for these patients (2).

Histological examination. Resected specimens were immediately fixed in $10 \%$ buffered formalin solution for at least $24 \mathrm{~h}$ at room temperature, and stained with hematoxylin and eosin. Histological specimens were subsequently cut into parallel sections 2-3 mm thick. Pathological specimens were assessed for resection margin status and histological characteristics by a single experienced pathologist. Tumor size was defined as the maximum tumor diameter on the original pathology report.

The degree of submucosal invasion was determined according to the Kudo classification (Fig. 1) (14). Specifically, the degree of submucosal invasion was classified into three categories based on invasion depth in surgically resected specimens: Infiltration into the upper third (sm1), middle third (sm2) and lower third (sm3) of the submucosal layer. In endoscopically resected specimens, the resected submucosal layer was vertically divided into two components. Submucosal invasion within the upper layer was regarded as sm1, invasion of the deeper layer was regarded as $\operatorname{sm} 2$ and a vertically positive margin was regarded as sm3 (15). Furthermore, sm1 cases were subclassified into smla, smlb, and sm1c based on the horizontal extension of the submucosally invaded area. Smla and smlb carcinomas were defined as slightly invasive, while sm1c, sm2, and sm3 carcinomas were considered massively invasive submucosal carcinomas.

Histological type was based on the World Health Organization Classification of Tumors (16). Tumors were examined histochemically using Victoria blue (Muto Pure Chemicals Co., Ltd., Tokyo, Japan) staining for vascular invasion and D2-40 (Dako North America, Inc., Carpinteria, CA, USA) expression for lymphatic invasion. Haematoxylin and eosin staining was also performed. Tumor budding was defined as an isolated single carcinoma cell or a cluster composed of $<5$ cells. After choosing one field in which budding was the most intense, a budding count was conducted in a field measuring $0.785 \mathrm{~mm}^{2}$ using an objective lens (magnification, $x 20$ ). A field with $\geq 5$ buds was regarded as grade 2 or 3 (17).

Statistical analysis. Nominal and ordinal variables are expressed as frequencies and percentages. Continuous variables are reported as mean \pm standard deviation (SD) and were compared using Student's $t$-test, whereas dichotomous variables were compared using $\chi^{2}$ or Fisher exact tests, as appropriate. All statistical analyses were performed using $\mathrm{R}$ ver. 2.10.0 (R Foundation for Statistical Computing, Vienna, Austria). All $\mathrm{P}$-values were two sided and $\mathrm{P}<0.05$ was considered to indicate a statistically significant difference.

\section{Results}

Patient population. A total of 19,864 patients with colorectal neoplasms underwent endoscopic or surgical tumor resection at the Showa University Northern Yokohama Hospital (Yokohama, Japan) between April 2001 and March 2013 (Fig. 2). Of these, 856 (4.3\%) patients exhibited colorectal T1 carcinoma. However, 299 patients with colorectal T1 carcinoma were excluded: 268 underwent endoscopic resection alone, 17 had synchronous advanced colorectal carcinoma, 3 had Lynch syndrome, 1 had inflammatory bowel disease and 10 were excluded for other reasons. Thus, the final study cohort consisted of 557 patients with colorectal $\mathrm{T} 1$ carcinoma, including 457 with colon carcinoma and 100 with rectal carcinoma. Of these, 293 patients (233 with colon and 60 with rectal carcinoma) underwent first-line surgery and lymph node dissection and 264 (224 with colon and 40 with rectal tumors) underwent first-line endoscopic resection followed by additional surgery. The total cohort of 557 patients included 210 women (37.7\%) and 347 men (62.3\%), among whom the mean $( \pm \mathrm{SD})$ age was $64.9 \pm 11.4$ years.

The median number of lymph nodes dissected per patient was 13 (range, 1-53). The median number of lymph nodes in cases of colon carcinoma was 13 (range, 1-53), while the median number of lymph nodes in cases of rectal carcinoma was 13 (range, 2-41). Of the 557 patients, 54 (9.7\%) exhibited evidence of lymph node metastasis. 
SMs

(Slightly invasive submucosal carcinoma)

\section{$\mathrm{SMm}$}

(Massively invasive submucosal carcinoma)
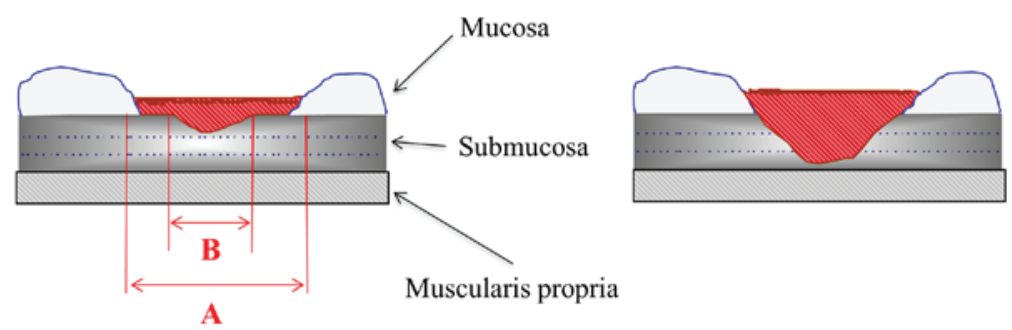

sml: upper $1 / 3$

smla: B/A $-1 / 4$ smlb: B/A $1 / 4-1 / 2$ SMs

smlc: B/A 1/2-

sm2: middle $1 / 3$

sm3: lower $1 / 3$

SMm

Figure 1. Classification of the degree of submucosal invasion. Submucosal invasion was scored according to the Kudo classification (14). SMs, slightly invasive submucosal carcinoma; SMm, massively invasive submucosal carcinoma.

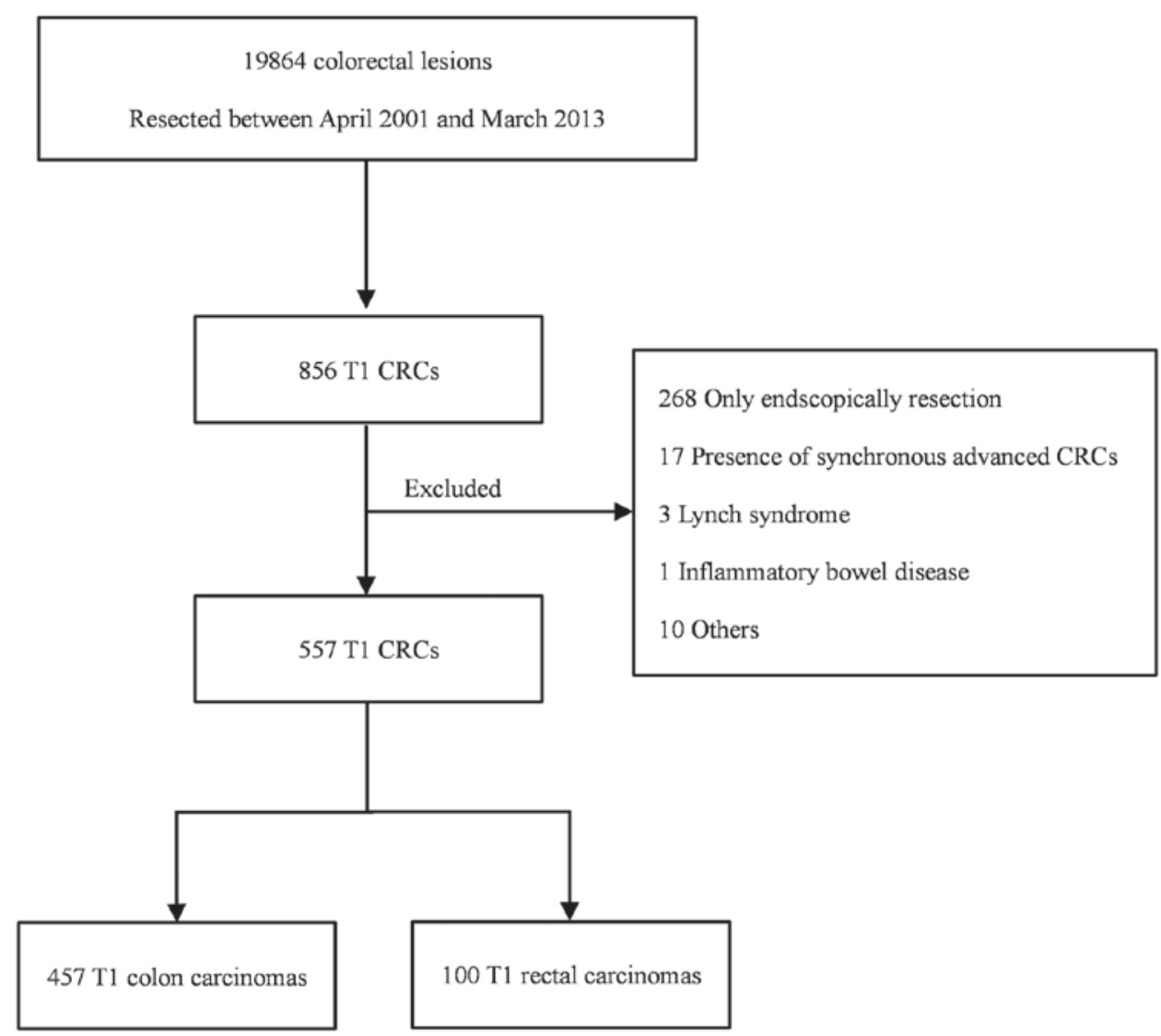

Figure 2. Patient flow chart demonstrating the potential pool of patients, and the inclusion criteria for patients eventually included in the study. CRC, colorectal carcinoma.

Clinicopathological features of colon and rectal T1 carcinoma. Table I presents the clinicopathological characteristics of the patient groups. Rectal T1 carcinomas were significantly larger $(23.7 \pm 13.1 \mathrm{~mm}$ vs. $19.9 \pm 11.0 \mathrm{~mm}, \mathrm{P}<0.01)$ and exhibited significantly higher rates of vascular invasion $(48.0 \%$ vs. $30.2 \%, \mathrm{P}<0.01)$ than colon $\mathrm{T} 1$ carcinomas.
The rates of lymphatic invasion ( $46.0 \%$ vs. $41.4 \%, \mathrm{P}=0.43$ ) and lymph node metastasis $(9.0 \%$ vs. $9.8 \%, \mathrm{P}=0.80)$ were similar in patients with rectal and colon $\mathrm{T} 1$ carcinomas. None of the other clinicopathological factors assessed differed significantly between patients with colon and rectal $\mathrm{T} 1$ carcinomas. 
Table I. Clinicopathological characteristics of patients with colon and rectal T1 carcinoma.

\begin{tabular}{|c|c|c|c|}
\hline Characteristic & Colon $(n=457)$ & Rectum $(n=100)$ & P-value \\
\hline Age, years \pm SD & $65.3 \pm 11.4$ & $62.9 \pm 10.8$ & 0.06 \\
\hline Males, n (\%) & $285(62.5)$ & $62(62.0)$ & 0.96 \\
\hline Tumor size, $\mathrm{mm} \pm \mathrm{SD}$ & $19.9 \pm 11.0$ & $23.7 \pm 13.1$ & $<0.01^{\mathrm{a}}$ \\
\hline Invasion depth, SMm (\%) & $422(92.3)$ & $93(93.0)$ & 0.99 \\
\hline Histological type, por or muc $(\%)$ & $89(19.5)$ & $15(15.0)$ & 0.33 \\
\hline Vascular invasion, $+(\%)$ & $138(30.2)$ & $48(48.0)$ & $<0.001^{\mathrm{a}}$ \\
\hline Lymphatic invasion, + (\%) & $189(41.4)$ & $46(46.0)$ & 0.43 \\
\hline Tumor budding, grade 2 or $3(\%)$ & $123(26.9)$ & $32(32.0)$ & 0.33 \\
\hline Lymph node metastasis, + $(\%)$ & $45(9.8)$ & $9(9.0)$ & 0.94 \\
\hline
\end{tabular}

${ }^{\mathrm{a}} \mathrm{P}<0.05$; Results are expressed as mean \pm standard deviation or number of patients (\%), as appropriate. SMm, massively invasive submucosal carcinoma; por or muc, poorly differentiated adenocarcinoma or mucinous carcinoma.

Table II. Morphology and tumor size according to LST subtypes of colon and rectal carcinoma.

\begin{tabular}{|c|c|c|c|}
\hline Characteristic & Colon $(n=457)$ & Rectum (n=100) & P-value \\
\hline Morphology & & & 0.10 \\
\hline Depressed, n (\%) & $129(28.2)$ & $36(36.0)$ & \\
\hline Flat, n $(\%)$ & $120(26.3)$ & $30(30.0)$ & \\
\hline Protruded, n (\%) & $208(45.5)$ & $34(34.0)$ & \\
\hline LST, n (\%) & $112(24.5)$ & $26(26.0)$ & 0.85 \\
\hline LST-G, n (\%) & $32(7.0)$ & $22(22.0)$ & $<0.01^{\mathrm{a}}$ \\
\hline Tumor size $\pm \mathrm{SD}, \mathrm{mm}$ & $41.1 \pm 18.1$ & $38.1 \pm 16.4$ & 0.54 \\
\hline LST-NG, n (\%) & $80(17.5)$ & $4(4.0)$ & $<0.01^{\mathrm{a}}$ \\
\hline Tumor size $\pm \mathrm{SD}, \mathrm{mm}$ & $24.4 \pm 9.5$ & $26.3 \pm 4.9$ & 0.70 \\
\hline
\end{tabular}

${ }^{a} \mathrm{P}<0.05$; Results are expressed as mean \pm standard deviation or number of patients (\%), as appropriate. LST, laterally spreading tumors; LST-G, granular-type LST; LST-NG, nongranular-type LST.

Morphology and size of colon and rectal T1 carcinomas. Table II presents comparisons between the morphology and size of colon and rectal T1 carcinomas. Morphologically, $28.2 \%(129 / 457)$ and $36.0 \%$ (36/100), of colon and rectal T1 carcinomas were depressed, respectively, 26.3\% (120/457) and $30.0 \%$ (30/100) were flat and $45.5 \%$ (208/457) and $34.0 \%$ $(34 / 100)$ were protruded. There were no significant differences in tumor morphology between the groups $(\mathrm{P}=0.10)$. In addition, $24.5 \%(112 / 457)$ of colon and $26.0 \%(26 / 100)$ of rectal T1 carcinomas were LSTs. LST-Gs accounted for $7.0 \%$ (32/457) of colon and $22.0 \%$ (22/100) of rectal T1 carcinomas, with mean tumor sizes of $41.1 \pm 18.1 \mathrm{~mm}$ and $38.1 \pm 16.2 \mathrm{~mm}$, respectively $(\mathrm{P}=0.54)$. $\mathrm{LST}-\mathrm{NG}$ accounted for $17.5 \%(80 / 457)$ of colon and $4.0 \%(4 / 100)$ of rectal T1 carcinomas, with mean tumor sizes of $24.4 \pm 9.5 \mathrm{~mm}$ and $26.3 \pm 4.9 \mathrm{~mm}$, respectively $(\mathrm{P}=0.70)$. However, colon $\mathrm{T} 1$ carcinomas classified as LST-Gs were significantly larger than those classified as LST-NGs $(41.1 \pm 18.1 \mathrm{~mm}$ vs. $24.4 \pm 9.5 \mathrm{~mm}, \mathrm{P}<0.01)$. In addition, rectal T1 carcinomas classified as LST-Gs were significantly larger than rectal tumors classified as LST-NGs $(38.1 \pm 16.4 \mathrm{~mm}$ vs. 26.3 $\pm 4.9 \mathrm{~mm}, \mathrm{P}<0.01)$.

\section{Discussion}

The present study investigated the clinicopathological differences between $\mathrm{T} 1$ rectum and colon carcinomas to assess whether they should be classified as a single entity or two distinct entities when considering the indications for surgical resection. Colon and rectal carcinoma share many features, however, they exhibit important clinicopathological and genetic differences (3). The results of the current study include three important clinical observations: i) The rates of lymph node metastasis were similar in rectal and colon $\mathrm{T} 1$ carcinomas; ii) rectal $\mathrm{T} 1$ carcinomas were generally larger than colon $\mathrm{T} 1$ carcinomas; iii) rectal $\mathrm{T} 1$ carcinomas were accompanied by significantly higher rates of vascular invasion than colon T1 carcinomas.

Lymph node metastasis is an important prognostic factor in patients with rectal and colon and T1 carcinoma and affects disease management. Nodal involvement is associated with an increased risk of local recurrence and shorter overall and disease-free survival time (18-20). Lymph node metastasis generally occurs in approximately $10 \%$ of patients with T1 colorectal carcinoma $(8,9)$. In the clinical guidelines of the 
Japanese Society for Cancer of the Colon and Rectum (JSCCR), patients who undergo endoscopic resection for T1 colorectal carcinoma are considered to be at extremely low risk of developing lymph node metastasis if they have negative vertical margins, well or moderately differentiated adenocarcinoma, absence of vascular or lymphatic invasion, grade 0 or 1 tumor budding and submucosal invasion $<1,000 \mu \mathrm{m}$ (2). These patients should be monitored periodically, however, they should not require additional surgery. Patients with $\leq 1$ of these factors are considered to be high risk and surgery, including lymph node dissection, is recommended.

Surgery for rectal carcinoma is more invasive than surgery for colon carcinoma. Standard abdominoperineal resection for low rectal carcinoma leaves many patients with permanent stomas. Advanced anus-preserving low anterior resection and intersphincteric resection have become more common as treatments for lower rectal carcinoma that avoid colostomies (21). However, some anal function disorders and complications have been reported following such resections, reducing patient quality of life (8). Due to similar rates of lymph node metastasis in patients with colon and rectal T1 carcinoma, surgical indications following endoscopic resection should be similar. However, it has been reported that high-risk patients with rectal T1 carcinoma have a significantly higher risk of local recurrence than high-risk patients with colon T1 carcinoma treated with endoscopic resection alone (5). Although rectal surgery is more invasive than colon surgery, additional surgery involving dissection of the lymph nodes is recommended for high-risk patients with rectal $\mathrm{T} 1$ carcinoma.

The present study indicated that rectal $\mathrm{T} 1$ carcinomas were larger than colon $\mathrm{T} 1$ carcinomas. This may be explained by differences in their morphologies, particularly in terms of the percentage of cases classified as LST-Gs. LSTs typically extend laterally and circumferentially rather than vertically along the colonic wall (22) and are associated with a lower frequency of invasion than polypoid lesions of similar size. Although a similar proportion of colon and rectal $\mathrm{T} 1$ carcinomas were classified as LSTs $(24.5 \%$ vs. $26.0 \%, \mathrm{P}=0.80)$, LST-Gs accounted for a significantly higher proportion of rectal LSTs than colon LSTs [84.6\% (22/26) vs. $28.6 \%$ (32/112), $\mathrm{P}<0.01]$. In accordance with previous results (23), LST-Gs were observed to be significantly larger than LST-NGs $(39.9 \pm 17.4 \mathrm{~mm}$ vs. $24.5 \pm 9.3 \mathrm{~mm}, \mathrm{P}<0.01)$, which may have affected the differences between the size and growth patterns of colon and rectal T1 carcinomas. Among patients who underwent endoscopic resection alone during the same period, rectal T1 carcinomas were significantly larger than colon T1 carcinomas $(25.1 \pm 19.7 \mathrm{~mm}$ vs. $19.7 \pm 12.1 \mathrm{~mm}$, $\mathrm{P}=0.016)$.

In agreement with a previous study (5), the rate of vascular invasion was observed to be significantly higher in rectal than colon T1 carcinomas. Differences in vascular invasion may have resulted from differences between the anatomical features of the rectum and colon. The rectum is supplied by both the inferior mesenteric artery and the internal iliac artery, with veins from the rectum returning to the vena cava and portal vein. Although blood vessel density differs in the colon and rectum, its association with vascular invasion remains unclear. It has been reported that tumor location in the rectum is a significant independent risk factor for delayed bleeding following endoscopic submucosal dissection for colorectal neoplasms, potentially as a consequence of differences in blood vessel density (24). Guidelines from the National Comprehensive Cancer Network, the European Society for Medical Oncology and JSCCR identify vascular invasion as a risk factor for lymph node metastasis and recommend that such patients undergo surgery with lymph node dissection $(2,25,26)$. Additionally, vascular invasion is an independent predictor of distant recurrence and survival in all patients with colorectal carcinoma $(27,28)$, suggesting the need for surgery in patients with colon and rectal T1 carcinomas who exhibit vascular invasion. However, as reported in a meta-analysis performed by Bosch et al (9), lymphatic invasion is the most powerful predictor of lymph node metastasis, whereas vascular invasion is a weaker predictor. This may explain the absence of a significant difference between rates of lymph node metastasis for colon and rectal T1 carcinoma in the current study.

The present study had three primary limitations: i) It was a retrospective analysis of patients treated at a single center, although it did include a larger cohort of patients than in previous, similar studies (29-32). ii) The retrospective design of the current study may have introduced some selection bias. When evaluating the incidence of lymph node metastasis, only patients who had undergone surgery were included. Patients treated by endoscopic resection alone were excluded as the incidence of lymph node metastasis this group was not precisely assessed. iii) The pathological diagnosis or features of these patients were not re-evaluated. Therefore, a large-scale prospective trial is necessary to verify the current management strategy for colorectal $\mathrm{T} 1$ carcinoma.

In conclusion, the results of the present study clearly demonstrate that patterns of lymph node metastasis did not differ between rectal and colon T1 carcinomas, even though rectal $\mathrm{T} 1$ carcinomas were larger and accompanied by a significantly higher rate of vascular invasion. Surgery should be the first-line treatment for high-risk patients with colon or rectal T1 carcinoma, even though rectal surgery is comparatively more invasive. Although investigated lymph node metastasis and other clinicopathological characteristics were investigated in the current study, survival and recurrence should be considered in future work.

\section{Acknowledgements}

The authors wish to express their gratitude to all members of the Digestive Disease Center of Showa University Northern Yokohama Hospital.

\section{References}

1. Matsuda T, Marugame T, Kamo K, Katanoda K, Ajiki W and Sobue T; Japan Cancer Surveillance Research Group: Cancer incidence and incidence rates in Japan in 2002: Based on data from 11 population-based cancer registries. Jpn J Clin Oncol 38: 641-648, 2008.

2. Watanabe T, Itabashi M, Shimada Y, Tanaka S, Ito Y, Ajioka Y, Hamaguchi T, Hyodo I, Igarashi M, Ishida H, et al: Japanese Society for Cancer of the Colon and Rectum (JSCCR) Guidelines 2014 for treatment of colorectal cancer. Int J Clin Oncol 20: 207-239, 2015.

3. Frattini M, Balestra D, Suardi S, Oggionni M, Alberici P, Radice P, Costa A, Daidone MG, Leo E, Pilotti S, et al: Different genetic features associated with colon and rectal carcinogenesis. Clin Cancer Res 10: 4015-4021, 2004. 
4. Wang H, Wei XZ, Fu CG, Zhao RH and Cao FA: Patterns of lymph node metastasis are different in colon and rectal carcinomas. World J Gastroenterol 16: 5375-5379, 2010.

5. Ikematsu H, Yoda Y, Matsuda T, Yamaguchi Y, Hotta K, Kobayashi N, Fujii T, Oono Y, Sakamoto T, Nakajima T, et al: Long-term outcomes after resection for submucosal invasive colorectal cancers. Gastroenterology 144: 551-559; quiz e14, 2013.

6. Pommergaard HC, Gessler B, Burcharth J, Angenete E, Haglind E and Rosenberg J: Preoperative risk factors for anastomotic leakage after resection for colorectal cancer: A systematic review and meta-analysis. Colorectal Dis 16: 662-671, 2014.

7. Kakodkar R, Gupta S and Nundy S: Low anterior resection with total mesorectal excision for rectal cancer: Functional assessment and factors affecting outcome. Colorectal Dis 8: 650-656, 2006.

8. Kobayashi H, Mochizuki H, Morita T, Kotake K, Teramoto T, Kameoka S, Saito Y, Takahashi K, Hase K, Oya M, et al: Characteristics of recurrence after curative resection for T1 colorectal cancer: Japanese multicenter study. J Gastroenterol 46 : 203-211, 2011.

9. Bosch SL, Teerenstra S, de Wilt JH, Cunningham C and Nagtegaal ID: Predicting lymph node metastasis in pT1 colorectal cancer: A systematic review of risk factors providing rationale for therapy decisions. Endoscopy 45: 827-834, 2013.

10. The Paris endoscopic classification of superficial neoplastic lesions: Esophagus, stomach and colon: November 30 to December 1, 2002. Gastrointest Endosc 58 (Suppl 6): S3-S43, 2003.

11. Kudo S, Lambert R, Allen JI, Fujii H, Fujii T, Kashida H, Matsuda T, Mori M, Saito H, Shimoda T, et al: Nonpolypoid neoplastic lesions of the colorectal mucosa. Gastrointest Endosc 68 (Suppl 4): S3-S47, 2008.

12. Kudo S, Tamura S, Nakajima T, Yamano $H$, Kusaka $H$ and Watanabe H: Diagnosis of colorectal tumorous lesions by magnifying endoscopy. Gastrointest Endosc 44: 8-14, 1996.

13. Matsuda T, Fujii T, Saito Y, Nakajima T, Uraoka T, Kobayashi N, Ikehara H, Ikematsu H, Fu KI, Emura F, et al: Efficacy of the invasive/non-invasive pattern by magnifying chromoendoscopy to estimate the depth of invasion of early colorectal neoplasms. Am J Gastroenterol 103: 2700-2706, 2008.

14. Kudo S, Tamegai Y, Yamano H, Imai Y, Kogure E and Kashida H: Endoscopic mucosal resection of the colon: The Japanese technique. Gastrointest Endosc Clin N Am 11: 519-535, 2001.

15. Tsuruta $\mathrm{O}$, Toyonaga A, Ikeda $\mathrm{H}$, Tanikawa $\mathrm{K}$ and Morimatsu $\mathrm{M}$ : Clinicopathological study of superficial-type invasive carcinoma of the colorectum. Int J Oncol 10: 1003-1008, 1997.

16. Bosman FT, Carneiro F, Hruban RH and Theise ND: WHO Classification of Tumours of the Digestive System Lyon, France, 2010.

17. Ueno H, Mochizuki H, Hashiguchi Y, Shimazaki H, Aida S, Hase K, Matsukuma S, Kanai T, Kurihara H, Ozawa K, et al: Risk factors for an adverse outcome in early invasive colorectal carcinoma. Gastroenterology 127: 385-394, 2004.
18. Zenni GC, Abraham K, Harford FJ, Potocki DM, Herman C and Dobrin PB: Characteristics of rectal carcinomas that predict the presence of lymph node metastases: Implications for patient selection for local therapy. J Surg Oncol 67: 99-103, 1998.

19. Saclarides TJ, Bhattacharyya AK, Britton-Kuzel C, Szeluga D and Economou SG: Predicting lymph node metastases in rectal cancer. Dis Colon Rectum 37: 52-57, 1994.

20. Chok KS and Law WL: Prognostic factors affecting survival and recurrence of patients with pT1 and pT 2 colorectal cancer. World J Surg 31: 1485-1490, 2007.

21. Paty PB, Enker WE, Cohen AM and Lauwers GY: Treatment of rectal cancer by low anterior resection with coloanal anastomosis. Ann Surg 219: 365-373, 1994.

22. Kudo S: Endoscopic mucosal resection of flat and depressed types of early colorectal cancer. Endoscopy 25: 455-461, 1993.

23. Uraoka T, Saito Y, Matsuda T, Ikehara H, Gotoda T, Saito D and Fujii T: Endoscopic indications for endoscopic mucosal resection of laterally spreading tumours in the colorectum. Gut 55: $1592-1597,2006$

24. Terasaki M, Tanaka S, Shigita K, Asayama N, Nishiyama S, Hayashi N, Nakadoi K, Oka S and Chayama K: Risk factors for delayed bleeding after endoscopic submucosal dissection for colorectal neoplasms. Int J Colorectal Dis 29: 877-882, 2014

25. Labianca R, Nordlinger B, Beretta GD, Mosconi S, Mandalà M, Cervantes A and Arnold D; ESMO Guidelines Working Group: Early colon cancer: ESMO Clinical Practice Guidelines for diagnosis, treatment and follow-up. Ann Oncol 24 (Suppl 6): vi64-vi72, 2013.

26. Benson AB III, Bekaii-Saab T, Chan E, Chen YJ, Choti MA, Cooper HS, Engstrom PF, Enzinger PC, Fakih MG, Fenton MJ, et al: Localized colon cancer, version 3.2013: Featured updates to the NCCN Guidelines. J Natl Compr Canc Netw 11: 519-528, 2013

27. Brown CE and Warren S: Visceral metastasis from rectal carcinoma. Surg Gynecol Obstet 66: 611-621, 1938.

28. Messenger DE, Driman DK and Kirsch R: Developments in the assessment of venous invasion in colorectal cancer: Implications for future practice and patient outcome. Hum Pathol 43: 965-973, 2012.

29. Kikuchi R, Takano M, Takagi K, Fujimoto N, Nozaki R, Fujiyoshi $\mathrm{T}$ and Uchida $\mathrm{Y}$ : Management of early invasive colorectal cancer. Risk of recurrence and clinical guidelines. Dis Colon Rectum 38: 1286-1295, 1995.

30. Okuyama T, Oya $\mathrm{M}$ and Ishikawa $\mathrm{H}$ : Budding as a risk factor for lymph node metastasis in pT1 or pT2 well-differentiated colorectal adenocarcinoma. Dis Colon Rectum 45: 628-634, 2002.

31. Nascimbeni R, Burgart LJ, Nivatvongs S and Larson DR: Risk of lymph node metastasis in T1 carcinoma of the colon and rectum. Dis Colon Rectum 45: 200-206, 2002.

32. Sakuragi M, Togashi K, Konishi F, Koinuma K, Kawamura Y, Okada $\mathrm{M}$ and Nagai $\mathrm{H}$ : Predictive factors for lymph node metastasis in T1 stage colorectal carcinomas. Dis Colon Rectum 46: 1626-1632, 2003. 